\title{
Single-molecule and Single-cell Approaches in Molecular Bioengineering
}

\author{
Michael A. Nash*ab
}

\begin{abstract}
Protein sequences inhabit a discrete set in macromolecular space with incredible capacity to treat human disease. Despite our ability to program and manipulate protein sequences, the vast majority of protein development efforts are still done heuristically without a unified set of guiding principles. This article highlights work in understanding biophysical stability and function of proteins, developing new biophysical measurement tools and building high-throughput screening platforms to explore functional protein sequences. We highlight two primary areas. First, molecular biomechanics is a subfield concerned with the response of proteins to mechanical forces, and how we can leverage mechanical force to control protein function. The second subfield investigates the use of polymers and hydrogels in protein engineering and directed evolution in pursuit of new molecular systems with therapeutic applications. These two subdisciplines complement each other by shedding light onto sequence and structural features that can be used to impart stability into therapeutic proteins.
\end{abstract}

Keywords: Atomic force microscopy $\cdot$ Hydrogels $\cdot$ Molecular biomechanics · Protein engineering

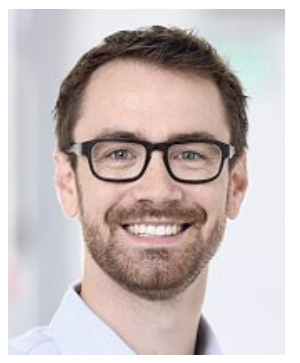

Prof. Dr. Michael Nash earned a Bachelor of Science in Cybernetics of Biomedical Systems from the University of California, Los Angeles in 2006 and a dual $\mathrm{PhD}$ in Bioengineering and Nanotechnology from the University of Washington, Seattle in 2010. Following Postdoctoral training in Applied Physics at Ludwig-Maximilians University of Munich, Nash began as tenuretrack Assistant Professor with joint appointment at the University of Basel, Department of Chemistry and ETH Zurich, Department of Biosystems Science \& Engineering in 2016. Nash's research is in the broad area of nanobiomaterials and biophysics, specifically focusing on single-molecule protein biomechanics, protein engineering, and the interface between synthetic and biological systems.

\section{Introduction}

\section{Protein Sequence Space}

Consider for a moment that the number of protein sequences 63 amino acids in length is greater than the number of atoms in the known universe $\left(10^{80}\right)$. Next consider that an average human protein has a length of approximately 450 amino acids. These simple calculations indicate that protein sequence space, although discrete, is unbelievably vast. And yet our ability to address medical challenges in the coming decades will depend on our capacity to efficiently search and characterize sequence and function within this space.

My research group at the University of Basel and ETH Zurich is motivated by this challenge to understand the functional space of protein sequences in pursuit of new therapies. I hope that by preparing this article for a general chemically literate audience as part of the 2020 'News from New Professors in Switzerland' Chimia special issue, I am able to introduce the chemical community in Switzerland and abroad to thematic areas we are actively pursuing in my lab, and provide a useful primer to those looking into these topics for the first time. By studying single-molecule un- folding and unbinding reactions using force microscopy, and utilize single-cell approaches to evolve new functions in therapeutic enzymes, we work at the information limit of living systems. I have split the content into two sections focusing on (1) molecular biomechanics, and (2) polymers and hydrogels in protein engineering.

\section{Protein Biopharmaceuticals}

The main application area of our single-cell and single-molecule engineering approaches is in biopharmaceuticals. Over the last 20 years, biological therapeutics have emerged as the fastest growing sector of the pharma industry, comprising over half of first-time approvals from 2015-2018, ${ }^{[1]}$ and representing close to $40 \%$ of approvals in 2019. [2] Biological therapeutics fall into several categories, the most prominent classes of which are monoclonal antibodies, in addition to other classes including antibody drug conjugates, therapeutic enzymes, peptides, vaccines in several formats, and small interfering RNA.

Whereas small molecule therapeutics consist of a few dozen atoms arranged in an atomically defined configuration lacking higher order structure, biologics consist of thousands of atoms that fold into complex 3D shapes that adopt a configuration belonging to a family of protein folds. Macromolecular therapeutics can be more specific in the body than small molecules, and can be artificially evolved in laboratory with genetic engineering, but enhanced specificity and evolvability come at a cost of ensuring stable folding during manufacturing, storage, and administration. Particularly the issues of protein folding, secondary and tertiary structural stability and post-translational modifications come into play. The three-dimensional structure of protein therapeutics is in fact not atomically defined but exists as an ensemble of conformations that are sampled by the molecule on the nanosecond timescale (Fig. 1).

\section{Part 1 - Molecular Biomechanics}

\section{Biophysical Stability at Equilibrium}

Developability refers to a range of properties including a biologic's feasibility of manufacture and storage, folding stabil- 


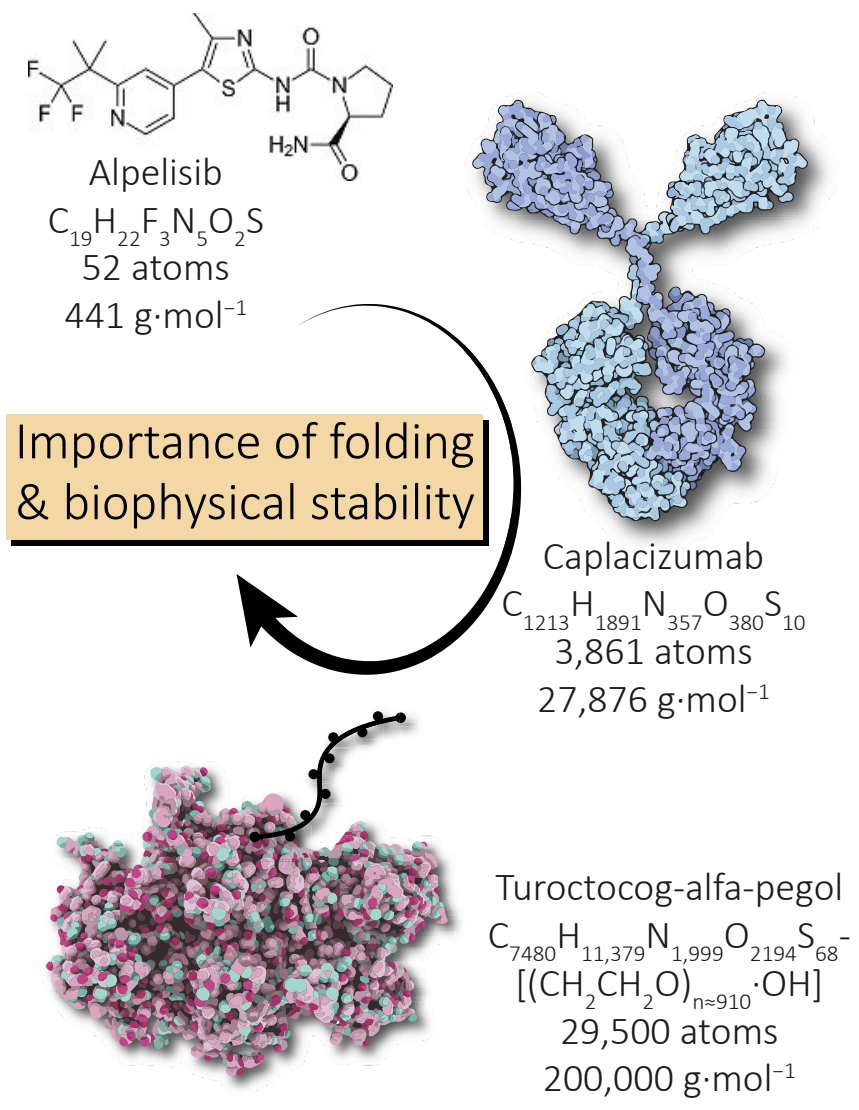

Fig. 1. Recently approved therapeutic compounds illustrating the transition from small molecule drugs to protein biopharmaceuticals. As molecular mass increases, atomic structures are no longer defined and structures sample an ensemble of conformations. This raises the importance of folding and biophysical stability as crucial in developing nextgeneration therapeutics.

ity, absence of non-specific interactions, colloidal stability and averseness to aggregation. Recent work demonstrated that biophysical stability is predictive of developability for a panel of monoclonal antibodies. ${ }^{[3]}$ Biophysical stability assays include equilibrium-based methods such as affinity-capture self-interaction nanoparticle spectroscopy (AC-SINS), clone self-interaction by bio-layer interferometry (CSI-BLI), and thermal melting temperature assessed by circular dichroism (CD) or nanoscale differential scanning fluorescence (nanoDSF). If a therapeutic scores poorly on one of these equilibrium-based stability assays, it is much more likely to fail in the pharmaceutical development pipeline. Biophysical stability assays are time-consuming, costly and consume large amounts of sample, and this failure mechanism wastes a significant amount of time and money during drug development. If we could develop improved methods for efficiently screening and characterizing biophysical stability of therapeutic compounds, we could accelerate this pipeline and rapidly contribute to next-generation therapies.

One of the limitations of the current paradigm is that biophysical screening is conducted at equilibrium. All the assays used for this purpose expose an ensemble of molecules to a uniform temperature and pressure, and determine the ratio of denatured to folded sample, or otherwise provide ensemble averages of the behavior of billions of molecules under equilibrium conditions. Living cells, tissues and organs, however, are notoriously non-equilibrium systems. Molecules can be acted upon through mechanical forces or chemical reactions requiring energy input to the molecules, and these factors are not well represented in equilibrium screens. Protein aggregation, for example, can be initiated from rare conformational states that are not populated at equilibrium. Furthermore, the manufacture of protein biopharmaceuticals in bioreactors occurs in environments that are far from equilibrium. For these reasons, we believe that studying non-equilibrium stability of biotherapeutics can provide unique insights into protein-based biopharmaceuticals.

\section{Single-molecule Mechanical Manipulation with the Atomic Force Microscope (AFM)}

Fluid cells for the atomic force microscope were introduced in $1989,{ }^{[4]}$ and the era of bio-AFM rapidly developed with the advent of single-molecule protein imaging under native conditions. ${ }^{[5,6]}$ Researchers discovered they could probe non-covalent protein and DNA interactions under force using functionalized AFM tips. ${ }^{[7-12]}$ Repetitive natural polyproteins such as smooth muscle Titin $^{[13,14]}$ or polyubiquitin chains ${ }^{[15,16]}$ with multi-domain structures were found to provide regularly spaced repeating saw-toothlike force-extension curves when stretched under tension. Each peak of the saw tooth corresponds to unfolding of a single domain within the polyprotein. This approach was utilized for generating artificial recombinant polyproteins comprising typically immunoglobulin domains that provided internal molecular controls for understanding mechanical unfolding of embedded domains of interest. Since these early developments, single-molecule AFM using polyproteins ${ }^{[17-23]}$ has provided detailed information about the relationships between secondary/tertiary structure and mechanical stability.

My lab has developed several methodological innovations ${ }^{[24-27]}$ for improving the throughput and data quality ${ }^{[18,28]}$ in single-molecule stretching experiments in order to gain insight into mechanostable receptor ligand interactions derived from adhesive bacteria. ${ }^{[29-31]}$ We have implemented molecular fingerprinting approaches for ensuring the validity of single-molecule receptor-ligand interactions (Fig. 2) whereby two domains with well-known unfolding patterns are fused with the receptor and ligand, respectively. Upon formation of the receptor-ligand complex and retraction of the cantilever, unfolding of the two fingerprint domains in series indicates successful loading of a singlecomplex. We have also implemented site-specific protein ligation chemistry based on a variety of enzymes, ${ }^{[24,26]}$ including Sfp, Sortase, or using isopeptide bond formation (Spytag/Spycatcher). These methods have allowed us to increase the statistical power of our analyses in characterizing mechanostable bacterial adhesion proteins, ${ }^{[27,30-33]}$ and therapeutic nanobodies. ${ }^{[34]}$

Protein mechanical stability is an important non-equilibrium stability parameter that can influence behavior in vivo. Mechanical stability describes how much tension a fold can bear before it unfolds, or how much force a receptor-ligand complex can withstand before breaking. Since force is a vector and not a scalar quantity, the mechanical response of a given protein strongly depends on the direction over which force is applied. Mechanically denaturing a protein by stretching it between its $\mathrm{N}$ - and $\mathrm{C}$-termini, for example, can give a very different response than stretching it between two internal residues. ${ }^{[35,36]}$ The field of molecular biomechanics is focused on understanding the influence of mechanical forces on biomolecular systems. We are interested in how molecules can sense and transmit force, and how mechanical forces can modulate protein activity. We classify molecular biomechanical studies into two general areas: native and artificial.

\section{Native Molecular Mechanical Systems}

Here we refer to molecular systems, typically protein-based, where force transduction plays an important role in physiology. Prominent examples include force-activated kinase enzymes found in muscle, ${ }^{[37-39]}$ or sound-sensitive tip-link proteins that generate signals in the inner ear. ${ }^{[40-44]}$ Another prominent example is the complex and highly regulated process of blood coagulation. ${ }^{[45-48]}$ Fibrin hydrogels which are the basic structural scaffold 

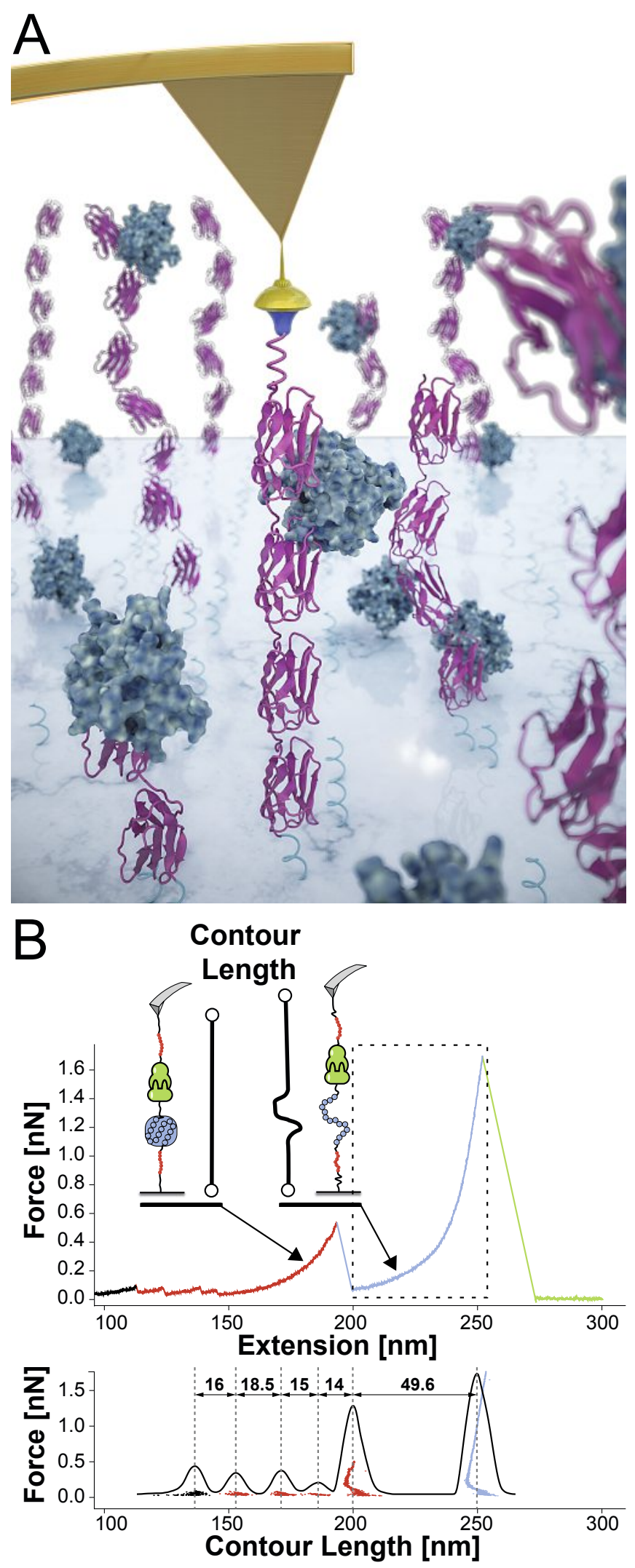

Fig. 2. Molecular biomechanics. (A) Single-molecule atomic force microscopy can be used to apply mechanical tension to individual protein domains and receptor-ligand complexes, and study unfolding and unbinding reactions under force. (B) Schematic of a typical data curve showing stretching of a single polyproteins, unfolding of fingerprint domains and rupture of receptor-ligand complexes. Non-linear entropic spring models of polymer elasticity are used to map the force-extension response into force-contour length space. This allows us to make domain assignments in the unfolding traces and understand which segments of the molecule are being mechanically denatured along the unfolding/unbinding reaction pathway. of blood clots exhibit strain-stiffening which is crucial for their role in preventing blood loss under contractile shear stress. ${ }^{[49,50]}$ Cell adhesion to the extracellular matrix is another mechanically regulated native process where integrins concentrate at focal adhesions and participate in complex mechanically-activated signaling. ${ }^{[51]}$ Mechanostable adhesion proteins is furthermore essential in bacterial pathogenesis. ${ }^{[52]}$ Bacteria are required to adhere to human tissues under shear stress, and have evolved adhesin proteins that exhibit force-activated binding ${ }^{[53]}$ to meet this challenge. Currently, the strongest known protein-protein interactions occur between these adhesins and human extracellular matrix proteins including fibrinogen and fibronectin. ${ }^{[5,55]}$

\section{Artificial Molecular Mechanical Systems}

Here we refer to systems where force is not a physiological parameter, but can be used to perturb an artificial system. Engineered artificial binding proteins can be developed to influence mechanical aspects of a target protein's physiology. Experimenters have used single-molecule mechanical manipulation to learn about proteins that have non-mechanical functions, and build synthetic mechanobiological systems. For example, single-molecule mechanical manipulation has been used to reveal the strength of metal coordination centers in proteins ${ }^{[56,57]}$ that otherwise have no mechanical role in vivo. Also, the mechanical activation of disulfide bond reduction reactions involving thiolated nucleophiles ${ }^{[58,59]}$ or reducing enzymes ${ }^{[60,61]}$ can be studied with single-molecule manipulation methods. Single-molecule cut-and-paste ${ }^{[62]}$ relying on a hierarchy of DNA dissociation forces in shear- or zip orientation has been used to assemble molecular patterns from the bottom-up. These studies indicate how mechanical forces can be used as an independent variable to accelerate chemical reactions that otherwise would not be observable on a realistic experimental timescale. This is closely related to the field of mechanochemistry, but here we are focused primarily on proteins and not synthetic polymeric materials.

As our understanding of molecular mechanics improves, we will see a more significant role for mechanopharmacology, which encompasses new therapeutic approaches that specifically target mechanical aspects of molecular and cellular (patho)physiology. In this direction, it is known for example that antibody therapeutics can dock and modulate the mechanical stability of the CD4 receptor domain required for HIV entry into cells. [63] Also, mechanical stability of immunoglobulin domains inversely correlates with their rates of nuclear pore complex translocation ${ }^{[64]}$ when fused with transcription factors, with implications on drug delivery or deliver of CRISPR-Cas therapeutics. For our part, our group is pursuing the application of mechanical forces to biopharmaceutical proteins as a method to characterize biophysical stability that can inform developability assessments in biopharma.

\section{Part 2 - Polymers and Hydrogels in Protein Engineering}

\section{Stimuli-responsive Synthetic Polymers}

Stimuli-responsive or 'smart' polymers are repetitive synthetic polymers that respond to small changes in environmental conditions by undergoing large changes in molecular conformation. ${ }^{[65]}$ Often times these materials lose solubility above a critical temperature, referred to as the lower critical solution temperature (LCST). Some of the most widely used synthetic smart polymers include poly(N-isopropylacrylamide) and poly(oligoethylene glycol methacrylate). These polymers can be readily polymerized from the surface of proteins by attaching ATRP initiators of RAFT agents and allowing polymerization to proceed from the protein surface in a 'grafting-from' synthetic approach, or alternatively site specifically conjugated to proteins near their active sites already as full-length polymers. ${ }^{[66]}$ Attachment near the ac- 
tive site and phase separation can be used to modulate protein function, such as switching enzyme activity in response to eternal stimuli. ${ }^{667,68]}$

However, synthetic polymer chemistry suffers from several limitations for therapeutics development. The polydispersity inherent in organic polymer synthesis results in a distribution of molecule lengths that could be problematic for clinical approval of therapeutics or drug delivery vehicles. Structure-function relationships are blurred when the sample is heterogeneous and tolerances for the molecular weight distribution need to be welldefined. To address this, protein-based polymers may be a valuable alternative.

\section{Protein-based Polymers}

Protein-based polymers are repetitive polymers comprising repeating segments of amino acids. They are genetically-encoded and produced in recombinant expression hosts, therefore completely monodisperse. Typically, protein-based polymers are either intrinsically disordered or adopt a weak helical conformation in aqueous solutions. A classical example is that of elastin-like polypeptides (ELPs) which comprise repeating (VPGXG) motifs where $X$ can take the identify of any amino acid except proline.

ELPs are artificial protein sequences derived from the native sequence of human tropoelastin, which is a highly flexible extracellular protein containing alternating hydrophilic and hydrophobic domains. In humans, there are 13 isoforms all derived from the $E L N$ gene by alternative splicing. The molecular weight of these individual isoforms ranges from approx. 50-70 kDa. Tropoelastin functions as the soluble precursor molecule of elastin, and after its expression in the cell it is secreted and forms aggregates that are cross-linked and incorporated into growing elastic fibers. With a half-life in the body of $>70$ years, elastin is highly stable and a fundamental component of the extracellular matrix $(\mathrm{ECM})$ of tissues whose function requires flexibility (e.g. blood vessels, heart, lung or skin). Elastin provides elasticity and strength and enables these tissues to return to their original shape after being stretched. Besides that, elastin is also involved in wound healing.

Originating from my $\mathrm{PhD}$ work at the University of Washington, Seattle, ${ }^{69-71]}$ my group continues to be active in developing new molecular systems incorporating both ELPs and synthetic smart polymers for applications in diagnostics, biomarker detections. We have implemented ELPs in single-molecule mechanical experiments to achieve uniform deformation and avoid mechanoisomerization reactions that are observed with PEG. ${ }^{[24]}$ In other recent work, we combined magnetic nanoparticles modified with poly(N-isopropylacrylamide) together with an alternative binding scaffold (Cohesin) that was fused with an ELP. By raising the temperature of the sample above the LCST of both the nanoparticles and the ELP-fusion protein, we found we were able to magnetically entrain and separate biomarkers bound to the Cohesin scaffold. This allowed us to perform biomarker separation and enrichment and to detect the target with higher sensitivity. ${ }^{[72]}$ In other work, we incorporated non-canonical amino acids into ELPs and used them as an assembly point for building multi-valent biosensing surfaces. This was innovative because multi-site non-canonical amino acid incorporation into ELPs had not yet been demonstrated, and was useful for increasing the binding capacity of the immunoassay surface. ${ }^{[73]}$

\section{Hydrogels for High-throughput Screening of Enzymes}

One large ongoing effort for us seeks to improve workflows for directed evolution by bringing in concepts from the fields of polymer and hydrogel engineering. Directed evolution is a laboratory method for improving phenotypic properties of proteins, and has been used extensively to enhance therapeutics enzymes for biopharmaceutical applications. ${ }^{[74]}$ Enzyme properties that can be enhanced by directed evolution include catalytic turnover rate, substrate binding affinity, substrate specificity/selectivity, as well as biophysical stability and can be conceived of as exploration of a multidimensional sequence/fitness landscape. ${ }^{[75]}$

Directed evolution essentially runs in an iterative process. In the first step, genetic diversity is generated by making mutations to the parent gene that encodes the enzyme. Many strategies can be used in designing and synthetizing gene libraries for directed evolution, for example, libraries may be based on error-prone PCR, homologous sequences, gene shuffling, and computational or rational design, as well as many other approaches. Following generation of genetic diversity, genes are transformed into an expression host such that each cell expresses only one variant from the library. Next, each variant must be screened or the host cells must be exposed to a selection pressure such that variants exhibiting enhanced phenotypic fitness survive. If a selection pressure cannot be developed that biochemically links enzyme activity with survival probability of the host, a screening approach is instead used which tests variants individually and ranks them in terms of phenotypic fitness under the screening condition. For typical screens, microbial colonies expressing a single variant would be inoculated into a 96-well plate and screened for enzymatic turnover using a colorimetric assay, or other suitable method for product quantification. Following the screening stage, the best performing variants can be combined and diversified, and the entire process is repeated in iterative rounds to improve the performance of the enzyme.

The screening stage is generally considered the bottleneck in directed evolution. The throughput of such screening campaigns is significantly limited by the requirement of achieving reaction compartmentalization to establish a genotype-phenotype linkage. The requirement of compartmentalizing individual clones using parallel multi-well cell culture and enzymatic turnover assays is laborious and slow. Typically, screening is done on cell lysates using robotic automation, which is complex, costly and achievable at a maximal rate of $\sim 10^{4}$ clones/week.

To address these significant barriers in enzyme directed evolution, we recently developed a hydrogel polymerization method $^{[76,77]}$ which provides yeast cells with an artificial phenotype that can be correlated with enzyme fitness. The reaction chemistry we developed allows yeasts to auto-encapsulate inside synthetic hydrogel capsules (Fig. 3) in response to enhanced activity of the target enzyme. The encapsulation reaction is dependent on the activity level of the target enzyme displayed on the outer cell wall. Exposure of cells to a reaction mixture containing macromonomer gel precursors along with the substrate of the enzyme initiates a reaction cascade that results in enzyme-mediated polymerization and cross-linking of a hydrogel capsule around individual cells. This approach offers a new genotype-phenotype linkage for directed evolution where one-pot reactions are completed in minutes and can be used to screen multimillion-member variant libraries under selective stress. We have several methods to perturb the enzyme variant library in order to screen for different molecular behaviors including folding stability, thermostability, catalytic turnover rate, or improved substrate binding affinity. This localized enzyme-mediated polymerization chemistry allows us to push the limits of laboratory directed evolution further, and combine with next-generation DNA sequencing technologies ${ }^{[78,79]}$ to understand sequence fitness landscapes at unprecedented scale.

In summary, I have provided a general overview for a chemically literate audience on the active themes in my group which concentrate on two areas: (1) molecular biomechanics of proteins and (2) polymers and hydrogels in protein engineering and directed evolution. These areas are connected in that they both seek to understand the relationships between sequence, structure and biophysical stability of therapeutics. The candidate molecules of interest for our investigations are primarily biotherapeutics, with 
a

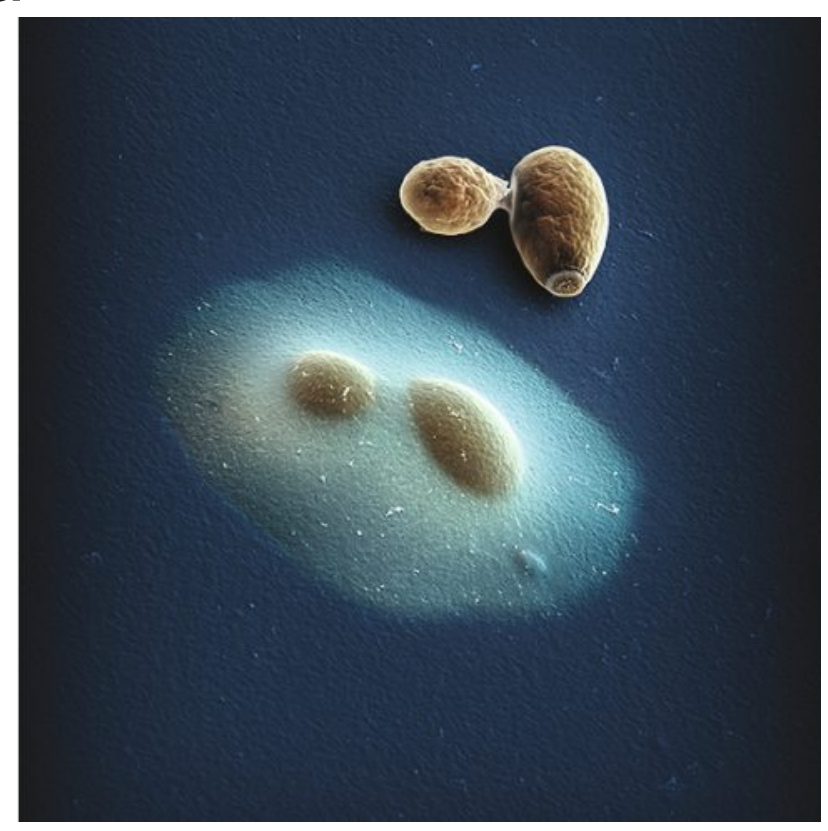

b

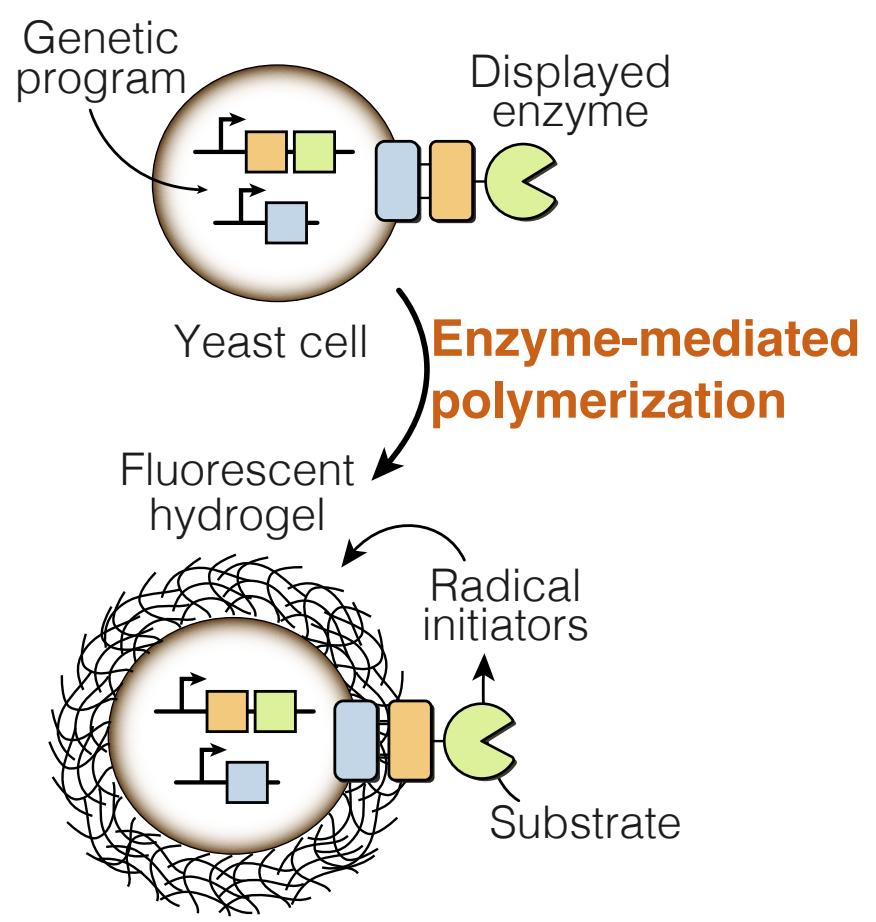

C

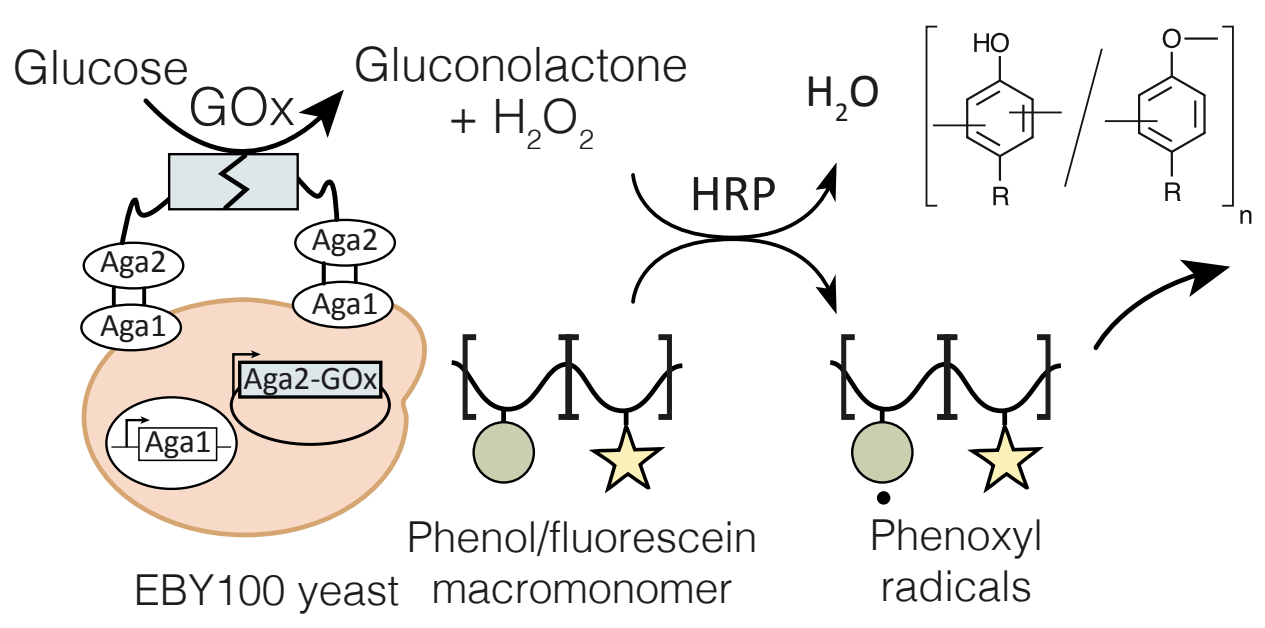

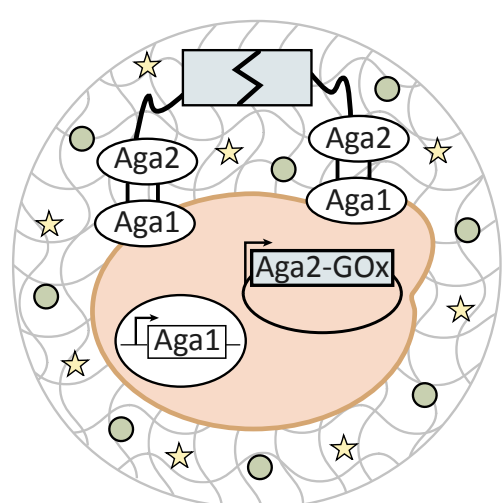

Single cell encapsulated in fluorescent hydrogel

Fig. 3. Localized hydrogel polymerization at the cell surface allows high-throughput screening of biocatalysts. (a) SEM image showing budding yeast encapsulated in an alginate hydrogel capsule that was dehydrated under SEM imaging conditions. (b) The concept behind such a screening approach is that enzymatic activity at the cell surface can localize hydrogel cross-linking reactions resulting in single-cell encapsulation. (c) Depiction of the reaction cascade for screening libraries of the FAD-dependent oxidoreductase enzyme Glucose oxidase (GOx). Variants encoding Gox dimers are displayed on the outer cell wall and catalyze oxidation of glucose to gluconolactone, producing peroxide. Horseradish peroxidase (HRP) takes up peroxide and catalyzes phenol cross-linking via a radical pathway. The short lifetimes of the radical species result in the cross-linking reaction being localized to the cell surface. Such an approach can be used for one-pot screening of large enzyme libraries for directed evolution.

a focus on alternative affinity proteins and binding scaffolds as well as therapeutic enzymes. In the future, advanced biophysical characterization methods and new approaches to high-throughput screening will allow us to generate vast datasets with paired sequence-function information. These will set the stage for datadriven protein engineering and advance our understanding of optimal design parameters for biotherapeutics.

Received: July 6, 2020

[1] G. Walsh, Nat. Biotechnol. 2018, 36, 1136

[2] C. Morrison, Nat. Biotechnol. 2020, 38, 126.

[3] T. Jain, T. Sun, S. Durand, A. Hall, N. R. Houston, J. H. Nett, B. Sharkey, B. Bobrowicz, I. Caffry, Y. Yu, Y. Cao, H. Lynaugh, M. Brown, H. Baruah, L. T.
Gray, E. M. Krauland, Y. Xu, M. Vásquez, K. D. Wittrup, Proc. Natl. Acad. Sci. USA 2017, 114, 944.

[4] B. Drake, C. B. Prater, A. L. Weisenhorn, S. A. Gould, T. R. Albrecht, C. F. Quate, D. S. Cannell, H. G. Hansma, P. K. Hansma, Science 1989, 243, 1586.

[5] D. J. Müller, Y. F. Dufrêne, Nat. Nanotechnol. 2008, 3, 261.

[6] M. Radmacher, R. W. Tillamnn, M. Fritz, H. E. Gaub, Science 1992, 257, 1900.

[7] V. T. Moy, E. L. Florin, H. E. Gaub, Science 1994, 266, 257.

[8] E.-L. Florin, V. T. Moy, H. E. Gaub, Science 1994, 264, 415

[9] E. L. Florin, M. Rief, H. Lehmann, M. Ludwig, C. Dornmair, V. T. Moy, H. E. Gaub, Biosens. Bioelectr. 1995, 10, 895 .

[10] A. Chilkoti, T. Boland, B. D. Ratner, P. S. Stayton, Biophys. J. 1995, 69, 2125.

[11] G. U. Lee, L. A. Chrisey, R. J. Colton, Science 1994, 266, 771.

[12] G. U. Lee, D. A. Kidwell, R. J. Colton, Langmuir 1994, 10, 354

[13] A. F. Oberhauser, P. K. Hansma, M. Carrion-Vazquez, J. M. Fernandez, Proc. Natl. Acad. Sci. USA 2001, 98, 468. 
[14] M. Rief, Science 1997, 276, 1109.

[15] M. Schlierf, H. Li, J. M. Fernandez, Proc. Natl. Acad. Sci. USA 2004, 101, 7299.

[16] M. Carrion-Vazquez, H. Li, H. Lu, P. E. Marszalek, A. F. Oberhauser, J. M. Fernandez, Nat. Struct. Biol. 2003, 10, 738

[17] M. L. Hughes, L. Dougan, Rep. Prog. Phys. 2016, 79, 076601.

[18] W. Ott, M. A. Jobst, C. Schoeler, H. E. Gaub, M. A. Nash, J. Struct. Biol. 2016, 197, 3.

[19] Y. Deng, T. Wu, M. Wang, S. Shi, G. Yuan, X. Li, H. Chong, B. Wu, P. Zheng, Nat. Commun. 2019, 10, 2775.

[20] H. Dietz, M. Bertz, M. Schlierf, F. Berkemeier, T. Bornschlögl, J. P. Junker, M. Rief, Nat. Protoc. 2006, 1, 80.

[21] T. Hoffmann, L. Dougan, Chem. Soc. Rev. 2012, 41, 4781.

[22] H. Dietz, M. Rief, Proc. Natl. Acad. Sci. USA 2006, 103, 1244.

[23] A. Valbuena, J. Oroz, R. Hervas, A. Manuel Vera, D. Rodriguez, M. Menendez, J. I. Sulkowska, M. Cieplak, M. Carrion-Vazquez, Proc. Natl. Acad. Sci. USA 2009, 106, 13791.

[24] W. Ott, M. A. Jobst, M. S. Bauer, E. Durner, L. F. Milles, M. A. Nash, H. E. Gaub, ACS Nano 2017, 11, 6346.

[25] C. Schoeler, T. Verdorfer, H. E. Gaub, M. A. Nash, Phys. Rev. E 2016, 94, 042412.

[26] W. Ott, T. Nicolaus, H. E. Gaub, M. A. Nash, Biomacromolecules 2016, 17, 1330

[27] M. A. Jobst, C. Schoeler, K. Malinowska, M. A. Nash, J. Vis. Exp. 2013, e50950.

[28] B. Yang, Z. Liu, H. Liu, M. A. Nash, Front. Mol. Biosci. 2020, 7, 85.

[29] M. A. Jobst, L. F. Milles, C. Schoeler, W. Ott, D. B. Fried, E. A. Bayer, H. E. Gaub, M. A. Nash, Elife 2015, 4, e10319.

[30] C. Schoeler, K. H. Malinowska, R. C. Bernardi, L. F. Milles, M. A. Jobst, E. Durner, W. Ott, D. B. Fried, E. A. Bayer, K. Schulten, H. E. Gaub, M. A. Nash, Nat. Commun. 2014, $5,1$.

[31] Z. Liu, H. Liu, A. M. Vera, R. C. Bernardi, P. Tinnefeld, M. A. Nash, 'High Force Catch Bond Mechanism of Bacterial Adhesion in the Human Gut', Nat. Commun. 2020, accepted.

[32] R. C. Bernardi, E. Durner, C. Schoeler, K. H. Malinowska, B. G. Carvalho, E. A. Bayer, Z. Luthey-Schulten, H. E. Gaub, M. A. Nash, J. Am. Chem. Soc. 2019, 141, 14752

[33] C. Schoeler, R. C. Bernardi, K. H. Malinowska, E. Durner, W. Ott, E. A. Bayer, K. Schulten, M. A. Nash, H. E. Gaub, Nano Lett. 2015, 15, 7370.

[34] H. Liu, V. Schittny, M. A. Nash, Nano Lett. 2019, 19, 5524.

[35] H. Dietz, F. Berkemeier, M. Bertz, M. Rief, Proc. Natl. Acad. Sci. USA 2006, 103, 12724

[36] D. J. Brockwell, E. Paci, R. C. Zinober, G. S. Beddard, P. D. Olmsted, D. A. Smith, R. N. Perham, S. E. Radford, Nat. Struct. Biol. 2003, 10, 731.

[37] E. M. Puchner, A. Alexandrovich, A. L. Kho, U. Hensen, L. V. Schäfer, B. Brandmeier, F. Gräter, H. Grubmüller, H. E. Gaub, M. Gautel, Proc. Natl. Acad. Sci. USA 2008, 105, 13385.

[38] S. W. Stahl, E. M. Puchner, A. Alexandrovich, M. Gautel, H. E. Gaub, Biophys. J. 2011, 101, 1978.

[39] F. Baumann, M. S. Bauer, M. Rees, A. Alexandrovich, M. Gautel, D. A Pippig, H. E. Gaub, Elife 2017, 6, DOI: 10.7554/eLife.26473.

[40] J. Oroz, A. Galera-Prat, R. Hervás, A. Valbuena, D. Fernández-Bravo, M. Carrión-Vázquez, Sci. Rep. 2019, 9, 13306.

[41] J. P. Hazra, A. Sagar, N. Arora, D. Deb, S. Kaur, S. Rakshit, Biochem. J. 2019, 476, 2411.

[42] M. Sotomayor, W. A. Weihofen, R. Gaudet, D. P. Corey, Nature 2012, 492 , 128.

[43] T. F. Bartsch, F. E. Hengel, A. Oswald, G. Dionne, I. V. Chipendo, S. S. Mangat, M. El Shatanofy, L. Shapiro, U. Müller, A. J. Hudspeth, Proc. Natl. Acad. Sci. USA 2019, 116, 11048.

[44] E. M. Mulhall, A. Ward, D. Yang, M. A. Koussa, D. P. Corey, W. P. Wong, Biorxiv DOI: $10.1101 / 763847$.

[45] A. Löf, P. U. Walker, S. M. Sedlak, S. Gruber, T. Obser, M. A. Brehm, M. Benoit, J. Lipfert, Proc. Natl. Acad. Sci. USA 2019, 116, 18798.

[46] J. P. Müller, S. Mielke, A. Löf, T. Obser, C. Beer, L. K. Bruetzel, D. A. Pippig, W. Vanderlinden, J. Lipfert, R. Schneppenheim, M. Benoit, Proc. Natl. Acad. Sci. USA 2016, 113, 1208.

[47] C. Baldauf, R. Schneppenheim, W. Stacklies, T. Obser, A. Pieconka, S. Schneppenheim, U. Budde, J. Zhou, F. Gräter, J. Thromb. Haemost. 2009, 7, 2096.
[48] J. Kim, C.-Z. Zhang, X. Zhang, T. A. Springer, Nature 2010, 466, 992.

[49] I. K. Piechocka, R. G. Bacabac, M. Potters, F. C. Mackintosh, G. H. Koenderink, Biophys. J. 2010, 98, 2281.

[50] K. A. Jansen, R. G. Bacabac, I. K. Piechocka, G. H. Koenderink, Biophys. J. 2013, 105, 2240.

[51] N. Strohmeyer, M. Bharadwaj, M. Costell, R. Fässler, D. J. Müller, Nat Mater. 2017, 16, 1262

[52] L. F. Milles, E. A. Bayer, M. A. Nash, H. E. Gaub, J. Phys. Chem. B 2017, $121,3620$.

[53] E. V. Sokurenko, V. Vogel, W. E. Thomas, Cell Host Microbe 2008, 4, 314.

[54] L. F. Milles, K. Schulten, H. E. Gaub, R. C. Bernardi, Science 2018, 359, 1527.

[55] P. Herman, S. El-Kirat-Chatel, A. Beaussart, J. A. Geoghegan, T. J. Foster, Y F. Dufrêne, Mol. Microbiol. 2014, 93, 356.

[56] P. Zheng, G. M. Arantes, M. J. Field, H. Li, Nat. Commun. 2015, 6, 7569.

[57] P. Zheng, C.-C. Chou, Y. Guo, Y. Wang, H. Li, J. Am. Chem. Soc. 2013, 135 17783.

[58] A. P. Wiita, S. R. K. Ainavarapu, H. H. Huang, J. M. Fernandez, Proc. Natl. Acad. Sci. USA 2006, 103, 7222.

[59] S. Garcia-Manyes, J. Liang, R. Szoszkiewicz, T.-L. Kuo, J. M. Fernández, Nat. Chem. 2009, 1, 236.

[60] T. B. Kahn, J. M. Fernández, R. Perez-Jimenez, J. Biol. Chem. 2015, 290, 14518.

[61] R. Perez-Jimenez, J. Li, P. Kosuri, I. Sanchez-Romero, A. P. Wiita, D. Rodriguez-Larrea, A. Chueca, A. Holmgren, A. Miranda-Vizuete, K. Becker, S.-H. Cho, J. Beckwith, E. Gelhaye, J. P. Jacquot, E. A. Gaucher, J. M. Sanchez-Ruiz, B. J. Berne, J. M. Fernandez, Nat. Struct. Mol. Biol. 2009, 16,890 .

[62] S. K. Kufer, E. M. Puchner, H. Gumpp, T. Liedl, H. E. Gaub, Science 2008 319, 594.

[63] R. Perez-Jimenez, A. Alonso-Caballero, R. Berkovich, D. Franco, M.W. Chen, P. Richard, C. L. Badilla, J. M. Fernandez, ACS Nano 2014, 8 , 10313.

64] E. Infante, A. Stannard, Board, Stephanie J., P. Rico-Lastres, E. Rostkova, A. E. M. Beedle, A. Lezamiz, Y. J. Wang, S. Gulaidi Breen, F. Panagaki, V. Sundar Rajan, C. Shanahan, P. Roca-Cusachs, S. Garcia-Manyes, Nat. Phys. 2019, 15, 973 .

[65] I. Cobo, M. Li, B. S. Sumerlin, S. Perrier, Nat. Mater. 2014, 14, 143.

[66] J. H. Ko, H. D. Maynard, Chem. Soc. Rev. 2018, 47, 8998.

[67] T. Shimoboji, E. Larenas, T. Fowler, S. Kulkarni, A. S. Hoffman, P. S Stayton, Proc. Natl. Acad. Sci. USA 2002, 99, 16592.

[68] V. Bulmus, Z. Ding, C. J. Long, P. S. Stayton, A. S. Hoffman, Bioconjug. Chem. 2000, 11, 78 .

[69] M. A. Nash, P. Yager, A. S. Hoffman, P. S. Stayton, Bioconjug. Chem. 2010, $21,2197$.

[70] M. A. Nash, J. N. Waitumbi, A. S. Hoffman, P. Yager, P. S. Stayton, ACS Nano 2012, 6, 6776 .

[71] M. A. Nash, J. J. Lai, A. S. Hoffman, P. Yager, P. S. Stayton, Nano Lett. 2010 10,85 .

[72] D. T. Ta, R. Vanella, M. A. Nash, Nano Lett. 2017, 17, 7932.

[73] D. T. Ta, R. Vanella, M. A. Nash, ACS Appl. Mater. Interfaces 2018, 10, 30147.

[74] C. Zeymer, D. Hilvert, Annu. Rev. Biochem. 2018, 87, 131.

[75] P. A. Romero, F. H. Arnold, Nat. Rev. Mol. Cell Biol. 2009, 10, 866.

[76] R. Vanella, D. T. Ta, M. A. Nash, Biotechnol. Bioeng. 2019, 116, 1878.

[77] R. Vanella, A. Bazin, D. T. Ta, M. A. Nash, Chem. Mater. 2019, 31, 1899

[78] P. A. Romero, T. M. Tran, A. R. Abate, Proc. Natl. Acad. Sci. USA 2015, 112 , 7159.

[79] M. S. Faber, T. A. Whitehead, Curr. Opin. Biotechnol. 2019, 60, 104.

\section{License and Terms}

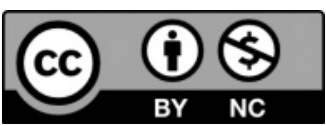

This is an Open Access article under the terms of the Creative Commons Attribution License CC BY_NC 4.0. The material may not be used for commercial purposes.

The license is subject to the CHIMIA terms and conditions: (http:// chimia.ch/component/sppagebuilder/?view=page \&id=12).

The definitive version of this article is the electronic one that can be found at https://doi.org/10.2533/chimia.2020.704 